\title{
Voluntary stuttering suppresses true stuttering: A window on the speech perception-production link
}

\author{
TIM SALTUKLAROGLU, JOSEPH KALINOWSKI, VIKRAM N. DAYALU, \\ ANDREW STUART, and MICHAEL P. RASTATTER \\ East Carolina University, Greenville, North Carolina
}

\begin{abstract}
In accord with a proposed innate link between speech perception and production (e.g., motor theory), this study provides compelling evidence for the inhibition of stuttering events in people who stutter prior to the initiation of the intended speech act, via both the perception and the production of speech gestures. Stuttering frequency during reading was reduced in 10 adults who stutter by approximately $40 \%$ in three of four experimental conditions: (1) following passive audiovisual presentation (i.e., viewing and hearing) of another person producing pseudostuttering (stutter-like syllabic repetitions) and following active shadowing of both (2) pseudostuttered and (3) fluent speech. Stuttering was not inhibited during reading following passive audiovisual presentation of fluent speech. Syllabic repetitions can inhibit stuttering both when produced and when perceived, and we suggest that these elementary stuttering forms may serve as compensatory speech gestures for releasing involuntary stuttering blocks by engaging mirror neuronal systems that are predisposed for fluent gestural imitation.
\end{abstract}

Pseudostuttering is the purposeful, voluntary, and controlled production of speech disruptions, such as repetitions and prolongations of speech sounds. These voluntary speech productions stand in stark contrast to the involuntary repetitions and prolongations of speech that are the fundamental characteristics of the disorder known as stuttering (Wingate, 1964). Although the ameliorative effects of pseudostuttering, or voluntary stuttering, on the true involuntary manifestations of stuttering are undeniable (Bloodstein, 1995; Van Riper, 1973), the underlying mechanism is not well understood. Early studies that examined immediate fluency enhancement via pseudostuttering yielded reductions in stuttering as high as $80 \%$ following the use of a form of pseudostuttering that was characterized by the production of syllabic repetitions (Fishman, 1937). This technique later became known in therapeutic circles as bouncing (Johnson, 1959) or clonic pseudostuttering (Van Riper, 1973). The high levels of immediate fluency enhancement attained suggest the potential to almost instantaneously inhibit true stuttering via the use of pseudostuttering.

Over the last century, the use of pseudostuttering has been readily integrated into standard therapeutic milieus as a secondary tool, with its ameliorative properties being

Correspondence concerning this article should be addressed to J. Kalinowski, Communication Sciences and Disorders, East Carolina University, Oglesby Drive, Greenville, NC 27858 (e-mail: kalinowskij@ mail.ecu.edu).

Note-This article was accepted by the previous editorial team, headed by Neil Macmillan. attributed to a reduction of the fear that is associated with stuttering. However, existing data do not support this explanation. People who stutter have not demonstrated an increase in autonomic responses considered to be associated with fear (e.g., skin conductance, heart rate, and pulse) during speech tasks (Janssen \& Kraaimaat, 1980; Peters \& Hulstijn, 1984), nor have any significant differences in self-reported levels of anxiety been reported in people who stutter (Blood, Blood, Bennett, Simpson, \& Susman, 1994; Kraaimaat, Janssen, \& van Dam-Baggen, 1991), as compared with nonstuttering counterparts. Furthermore, autonomic responses in people who stutter have failed to show marked decreases under conditions that reduce stuttering (Adams \& Moore, 1972; Gray \& Brutten, 1965; Reid \& Lingwall, 1976, 1980; Ritterman \& Reidenbach, 1975). These data clearly suggest that the behavioral manifestations of stuttering and the ameliorative effects of pseudostuttering are not directly linked to simple fear reduction. Given the potential for immediate fluency enhancement following the use of pseudostuttering, it is proposed that certain pseudostuttering patterns (e.g., the use of syllabic repetitions) possess inherent properties that immediately and effectively inhibit the occurrence of the involuntary stuttering block or moment.

Both Fishman (1937) and Meisner (1946) found that pseudostuttering using syllabic repetitions (e.g., tuh-tuhtuh-table) produced significant stuttering reductions in the ensuing speech. Fishman's (1937) study also showed that the voluntary emulation of other types of stuttering behaviors (such as prolonging sounds or silently blocking the production of speech sounds) proved to be relatively ineffective for fluency enhancement, suggesting the impor- 
tance of syllabic repetitions in the inhibition of stuttering. Furthermore, both Johnson (1959) and Van Riper (1973) favored the use of syllabic repetitions (e.g., bouncing, or clonic pseudostuttering) over the voluntary use of any other form of overt stuttering behavior in their therapeutic approaches. These therapeutic choices, as well as the empirical evidence presented, are compelling and suggest one conclusion: The active production of syllabic repetitions prior to speech can effectively enhance fluency in people who stutter by immediately inhibiting the involuntary stuttering block.

Stuttering inhibition via the production of pseudostuttering may be considered an active process, since it requires the active generation of the inhibitory agent by the speaker prior to the intended speech act. The possibility of actively inhibiting stuttering prior to speech was supported in a recent study in which stuttering was reduced by $30 \%$ following the production of a continuous vocalic/a/ (Dayalu, Saltuklaroglu, Kalinowski, Stuart, \& Rastatter, 2001). However, powerful passive stuttering inhibitors, such as choral speech, shadow speech, delayed auditory feedback, frequency altered feedback, and visual choral speech (Saltuklaroglu, Dayalu, \& Kalinowski, 2002), are known to immediately and effectively enhance fluency in those who stutter, without the generation of a prior inhibitory agent. Interestingly, Van Riper (1973) reported that stuttering could be passively reduced in young children by asking them to do no more than listen to a clinician producing easy syllabic repetitions, similar to those produced when syllabic repetitions are used in active pseudostuttering. Furthermore, anecdotal reports exist in which people who stutter have claimed that fluency enhancement has followed observation of stuttering in others. Both of these examples suggest the possibility of passively inhibiting stuttering prior to speech production via the presentation of syllabic repetitions. If the simple witnessing of another person producing easy syllabic repetitions is fluency enhancing to those who stutter, we suspect that the surfacing of observable stuttering behaviors may be a purposeful compensatory act for the occurrence of an involuntary block. Their production may target and induce changes to certain neurophysiological systems to overcome the involuntary block.

The question that arises is whether simply hearing and viewing someone else produce easy syllabic repetitions may serve as a stuttering inhibitorfor those who stutter. To the best of our knowledge, no empirical data exist to support this notion, yet such a finding would be consistent with contemporary theories of speech perception that claim that speech perception and production are merely "flipsides of the same coin" (Liberman \& Mattingly, 1985). If speech perception and production are as tightly interwoven as some believe, the perception of a speech act that inhibits stuttering prior to speech production, such as pseudostuttering, should possess ameliorative effects comparable to those that occur when that same act is actually produced. Evidence of this phenomenon might be substantiation of the link between speech perception and production benefiting a pathological population and, as such, may be a boon to those who stutter.

\section{METHOD}

\section{Participants}

Ten adults who stutter ( 9 males and 1 female) with a mean age of 24.5 years $(S D=6.8)$ participated in this study. The participants did not present with any other speech, language, or hearing disorders, and all had normal or corrected-to-normal vision. Each participant had a history of therapy, yet was not receiving any therapy at the time of this study. Informed consent (approved by the University and Medical Center Institutional Review Board, East Carolina University) was obtained from all the participants.

\section{Instrumentation}

All the stimuli sets were recorded onto a readable digital videodisc (DVD-R), using Apple IDVD software, and were played using a Philips DVD 701 player. The visual stimuli were presented to each participant with a Sony Glasstron PLM A-35 personal monitor. The audio signal was routed through a Mackie 1202 mixer and was presented to each participant at his or her most comfortable hearing level, using EAR Tone 3A insert headphones. The experimenter watched the video stimulus on a 21-in. Samsung color television and used the remote control unit for the DVD player to play and pause the DVD during the presentation of each set of tokens.

\section{Experimental Conditions}

Each participant read text aloud under a control condition and four experimental conditions. The control condition was the simple presentation of text tokens that the participant was asked to read without any additional stimulus prior to the reading of each target utterance. The experimental conditions consisted of audiovisual presentation of a woman speaking prior to the participant's reading a target utterance. The woman produced either pseudostuttered or fluent speech, and the participant was asked either to passively observe and listen to the woman or to actively shadow (directly imitate) the woman prior to reading the target utterance. Hence, the four experimental conditions were (1) passive pseudostuttering (PP), (2) passive fluent speech (PF), (3) active pseudostuttering (AP), and (4) active fluent speech (AF).

\section{Stimuli}

In order to create stimuli for the conditions requiring passive and active pseudostuttering, a normally fluent adult female was asked to read nonstandardized junior high school level passages that had previously been used in other published research (Dayalu et al., 2001; Kalinowski, Dayalu, Stuart, Rastatter, \& Rami, 2000). The woman read these passages, producing three relatively evenly spaced syllabic repetitions of approximately equal duration and stress on every word. Each syllabic repetition consisted of the neutral (schwa) vowel sound's being added to the first phoneme of every word, similar to the previously described method of bouncing (e.g., tuh-tuh-tuhtable) for consonant-initiated words. For words that were vowel initiated, she simply repeated the initial vowel three times before saying the word (e.g., a-a-a-and). Approximately $30 \%$ of the pseudostuttered stimuli were analyzed for their temporal characteristics. The mean duration of each syllabic repetition was found to be $0.28 \mathrm{sec}$ $(S D=0.08)$, and the mean duration of pauses following each syllabic repetition was $0.43 \mathrm{sec}(S D=0.08)$. Hence, the mean duration of the three syllabic repetitions prior to each word, including pauses between syllables, was $2.02 \mathrm{sec}(S D=0.21)$, and the resulting speech that included the syllabic repetitions and intersyllabic pauses had a mean rate of approximately 89 syllables per minute (SPM). The stimuli for the active and passive fluent speech conditions consisted of the woman's reading different nonstandardized junior high 
school level passages at a normal rate and using natural intonation patterns. The mean speech rate during fluent readings (not including pauses greater than $0.1 \mathrm{sec}$ ) was $217 \mathrm{SPM}$.

Digital audiovideo recordings were made of the woman's face while she was reading (pseudostuttering and fluent speech), using a Sony 537-A Betacam camera linked to a Sony DSR-40 DVCam recording deck. A Shure T1 wireless microphone transmitter and a Shure VP3-CV receiver were used to record the audio signal. At all times, the woman was looking directly at the camera, with her lips and mouth in clear view. Individual tokens were then constructed for the pseudostuttered and fluent experimental conditions, using Adobe Premier 6.0 software. The digital recordings were broken down into segments of 4-7 syllables (1-4 words). Following each audiovideo clip of the woman pseudostuttering or producing fluent speech, a 2-sec video clip of lexically different (4-7 syllables) junior high school level text was inserted after each segment that would be the target utterance for the participants to read aloud. This text was displayed in a black font at the center of a white screen, making it clearly legible to each participant. Thus, one token of stimulus was the audiovisual presentation of pseudostuttered (e.g., "thuh-thuhthuh-the ruh-ruh-ruh-radio wuh-wuh-wuh-was") or fluent (e.g., "today we receive") speech, followed by the 2-sec appearance of lexically different text that was approximately matched for the number of syllables with the audiovisual presentation of the woman speaking. For each stimulus set, 60-65 tokens were created until 250 syllables of text were generated for the participants to read. Stimulus tokens for the control condition were constructed in a similar fashion as those for the experimental conditions but consisted only of text (i.e., without the audiovisual presentation of the woman speaking prior to the display of each token).

In order to minimize experimental effects due to stimulus bias, three different pseudostuttered, fluent, and control stimulus sets were created with the above procedure. Hence, nine stimulus sets were created in total. The tokens for the experimental conditions were all constructed so that the pseudostuttered or fluent speech differed lexically from the text that was to be read by the participant.

\section{Design and Procedure}

A potential shortcoming of an experimental design that requires people who stutter to produce pseudostuttered or fluent speech is a possible contamination by true stuttering that may or may not be overtly detectable (Armson \& Kalinowski, 1994). One way to combat such a problem is to make use of a potent natural fluency enhancer, such as shadowed (directly imitated) speech, in order to generate stutter-free pseudostuttered or fluent speech. Shadowing has repeatedly been shown to be immediately fluency inducing in people who stutter (Cherry \& Sayers, 1956; Healey \& Howe, 1987; Kondas, 1967) and has substantially reduced the potential for contamination by true stuttering in this procedure.

For each of the experimental conditions, the participants were asked to read text that was displayed on the screen of the Sony Glasstron at their normal speech rates and without using any therapeutic techniques that may have been previously learned to reduce stuttering. In the control condition, the participants were simply asked to read the text as it was displayed on the screen of the Sony Glasstron. In the passive pseudostuttered condition, the participants were asked only to watch and listen to the woman producing pseudostuttered speech and then immediately read the text that followed on the screen of the Sony Glasstron. They were specifically instructed not to reproduce any of the articulatory speech movements made by the woman while she was speaking. The instructions and procedure for the passive fluent condition were similar to the passive pseudostuttered condition, except that the woman was producing fluent rather than pseudostuttered speech. In the active pseudostuttered condition, each participant was asked to begin shadowing the pseudostuttered utterance produced by the woman as soon as she produced the first syllable, to maintain shadowing throughout the woman's utterance, and then to immediately read the text that followed. The ac- tive fluent condition also included active shadowing of the woman's fluent speech, followed again by reading of the displayed text. The instructions and procedure for the active fluent condition were similar to those for the active pseudostuttered condition.

For every condition, as each new token of text appeared on the screen, the experimenter paused the playing of the DVD, giving the participant sufficient time to read the text. For each participant, two of the three pseudostuttered and two of the three fluent stimulus sets were randomly selected for the two pseudostuttered and two fluent conditions, yet no participant received the same stimulus set for more than one experimental condition. In addition, one of the three control stimulus sets was randomly selected for the control condition, and the presentation order of each of the five conditions (one control and four experimental) was randomized.

Stuttering was defined as part-word repetitions, audible prolongations, and inaudible articulatory postural fixations (e.g., silent blocking on speech sounds). Frequency counts of stuttered syllables were collected while recorded videotapes of the participants under each condition were observed. The first 250 syllables of data were used in the analysis for each condition. The same research assistant and a second trained research assistant recalculated the number of stuttered words for $10 \%$ of the samples chosen at random. Interjudge word-by-word agreement, as indexed by Cohen's (1960) kappa, was .89. Intrajudge Cohen's kappa word-by-word agreement was .91. Kappa values above .75 represent excellent agreement (Fleiss, 1981). The data were analyzed using a one-factor, five-level repeated measure analysis of variance, with $p$ values less than .05 considered to be statistically significant.

\section{RESULTS}

The means and standard errors for the frequency of stuttered syllables per 250 syllables read for each condition are as follows: (1) control condition, $M=51.60(S E=$ 11.88), (2) PP condition, $M=32.20(S E=9.96)$, (3) $\mathrm{PF}$ condition, $M=42.50(S E=11.03)$, (4) AP condition, $M=30.40(S E=9.88)$, and (5) AF condition, $M=29.80$ $(S E=8.64)$. The stuttering frequency in the control condition was compared with those in the four experimental conditions. A one-factor repeated measure analysis of variance revealed a significant main effect of the experimental conditions on stuttering frequency $[F(4,36)=$ $\left.5.65, p<.01, \eta^{2}=.39, \Omega=.96\right]$.

To examine the differential effects of the experimental conditions on levels of stuttering inhibition and provide answers to the questions posed, a series of planned single- $d f$ contrasts were conducted (Keppel, 1991). A significant difference was found between the passive PP and PF conditions $(p=.04)$. However, no significant difference was found between the AP and AF conditions $(p=.92)$. Also, the PP condition was not found to be significantly different from the active AP and AF conditions ( $p=.54)$. Finally, there was no significant difference between the control and PF conditions $(p=.15)$. From these contrasts, it also may be logically inferred that the PP, AP, and AF conditions were all significantly different from control and $\mathrm{PF}$ conditions.

\section{DISCUSSION}

This study provides the first compelling evidence that the link between speech production and speech perception 
can benefit a pathological population. The most salient finding is that prior to speech production, stuttering can be inhibited via the external presentation of voluntarily produced syllabic repetitions. Simply put, stuttering can be passively inhibited prior to speaking by having those who stutter merely watch and listen to another person producing easy syllabic repetitions. This passive inhibitory effect occurred to a extent (approximately 40\%) similar to that found when those same syllabic repetitions were actively and mechanically produced. Therefore, the data herein validate a phenomenon often self-reported by those who stutter: that simply listening to stuttering being produced by others who stutter inhibits overt stuttering events in themselves.

Interestingly, both active conditions also produced similar levels of fluency enhancement, although they required the production of notably different utterances prior to target reading. However, upon closer examination, both conditions may be essentially considered to be the same, since both involved shadowing, a permutation of choral speech that has consistently been found to increase fluency levels (Cherry \& Sayers, 1956; Healey \& Howe, 1987; Kondas, 1967). The use of shadowed speech created a relatively stutter-free active speech act before the participant was required to read the intended production in both the active conditions. Thus, the approximately $40 \%$ decrease in stuttering found in both active conditions may be a direct result of carryover to the ensuing reading from the enhanced fluency obtained during shadowing (Dayalu et al., 2001), irrespective of the content (i.e., either fluent or pseudostuttered) of the shadowed component. Stated concisely, in people who stutter, fluency begets fluency. Furthermore, fluency is both transitory and transferential, meaning that people who stutter appear to be capable of generating an increased proportion of fluent utterances for a short period following the removal of a fluency-enhancing agent.

The unifying factor in the three conditions (PP, AP, and $\mathrm{AF}$ ) that resulted in approximately $40 \%$ fluency enhancement is the repetition of speech gestures - perceived (PP), produced (AF), or perceived and produced (AP) - that is also found in such fluency-enhancing conditions as choral or shadowed speech. A neuronal substrate that may be responsible for the fluency enhancement provided by the perception or production of repetitive speech gestures is the mirror neuronal system. Mounting evidence supports the presence of mirror neurons in portions of the frontal lobe, including Broca's motor area, that have been found to fire both when goal-directed actions or gestures are observed and when they are produced (Kohler et al., 2002; Rizzolatti \& Arbib, 1998). These neurons are thought to play a key role in the fluent imitation of motoric gestures, including speech (Skoyles, 1998), such that they may predominate over the initial phases of cognitive and linguistic development, during which gestural imitation plays an integral role. It may not be coincidental that stuttering generally does not develop in children until the termination of this initial imitative phase (Kalinowski \& Saltuklaroglu, 2003). The existence of a neuronal system that can be ac- tivated by either repeated observations or productions of speech gestures and allows for their fluent imitation explains why in the PP condition, in which the same syllabic repetitions that were perceived in triplicate, fluency was enhanced to the same extent as in the AP and AF conditions, in which the imitated forms were actively produced. In other words, repetitive speech gestures, either perceived or produced, appear to centrally prime the speech-producing mechanism via the engagement of mirror neurons, a condition that may be especially beneficial in the inhibition of stuttering. From the arena of fluency enhancement, these data further support the notion that the mirror system provides the neuronal "strong link" (Rizzolatti \& Arbib, 1998) that unites speech production and perception according to the motor theory (Liberman \& Mattingly, 1985). Since these two processes may be one and the same at a neuronal level, it is not possible to determine, nor should it be a methodological concern, whether the syllabic repetitions were covertly imitated during the PP condition, during which the participants were specifically asked not to replicate any of the woman's articulatory movements. In fact, it is the repetitive nature of pseudostuttering that may trigger some covert gestural representation and facilitate the engagement of mirror neuronal systems.

In contrast, the passive fluent (PF) condition did not yield a statistically significant degree of fluency enhancement when compared with the control condition. Inherently, such a finding appears logical, for if simply watching and listening to fluent speech patterns produced by most people could serve as a fluency enhancer prior to the initiation of speech, stuttering would be much more easily inhibited during normal conversational speech. Normally, fluent speech is nonrepetitive by nature and probably not as easily amenable to covert repetition as pseudostuttered speech. For this reason, its simple perception prior to a target utterance does not appear to be sufficient to enable the inhibitory mechanism that appeared to be engaged when it was actively imitated via shadowing prior to target utterance. Although the pseudostuttered and fluent stimuli were linguistically matched for the number of syllables in the tokens, because of the differences in the nature of pseudostuttered and fluent speech (e.g., reduced speech rate due to intersyllabic pauses in pseudostuttered speech and the presence or absence of syllabic repetitions), in this study, they could not be temporally matched. To the best of our knowledge, no reports exist of stuttering's being reduced by the prior presentation of any quantity of normally fluent speech. As such, we suspect that the difference in length between the PF and the PP tokens did not significantly impact the results of this study.

Considering the possible role of the mirror system in fluency enhancement, it may not be surprising that syllabic repetitions appear to be the prevalent marker of the initial stages of developmental stuttering (Bloodstein, 1995). Before sound prolongations, postural fixations, and visible tensions manifest in children, stuttering is predominantly characterized by the presence of easy syllabic repetitions (Ambrose \& Yairi, 1999). These easy syllabic 
repetitions may be considered the most elementary form of stuttering and may represent the most primal or easily produced set of speech patterns or speech gestures for both children and adults who stutter. They have been described as being the "best compensatory mechanism" at the disposal of the speech motor system for overcoming the central involuntary stuttering block (Dayalu et al., 2001), a notion that is extended in this study to include an attempt to access the mirror system and regain fluent speech production (Kalinowski \& Saltuklaroglu, 2003). Thus, elementary repetitions of speech may represent a true telos or purpose for the production of discrete stuttering behaviors. By interpreting these overt behaviors as merely peripheral compensatory mechanisms for a centrally originating disorder, it becomes apparent that their frequency of manifestation will subside with the inhibition of the central involuntary block that is paramount to their surfacing. Syllabic repetitions appear to possess a fluencyenhancing property so powerful and unique that their active production is not even necessary for them to be effective as stuttering inhibitors prior to speech production. To our knowledge, no other pattern of speech has been found to possess these ameliorative properties when simply presented to a person who stutters prior to speaking.

In this experimental setting, shadowing was used in the active conditions to ensure that the active carrier stimulus would be produced fluently without the possibility of contamination from true stuttering (Armson \& Kalinowski, 1994). However, in clinical practice, the use of clonic pseudostuttering, or bouncing, is implemented without the use of a concomitant passive fluency-enhancing agent, since the syllabic repetitions have been found generally to be easily and fluently reproducible (Johnson, 1959; Van Riper, 1973). It has been suggested that in fluent speakers, a person's own speech may prime the auditory cortex, possibly helping to evoke the patterns of cortical activation and inhibition consistent with fluent speech production (Curio, Neuloh, Numminen, Jousmaki, \& Hari, 2000; Numminen \& Curio, 1999; Numminen, Salmelin, \& Hari, 1999). This cortical priming may be inherently compromised by stuttering blocks, and it is possible that the generation of the most elementary compensatory units of stuttering that can be fluently produced (via pseudostuttering) on a consistent basis engages mirror systems to temporarily inhibit the stuttering block. This may help normalize the system by producing patterns of cortical priming similar to those produced by fluent speakers.

Rather than being associated with simple fear reduction, the ameliorative effects of pseudostuttering appear to be linked to the production or perception of repeated syllables that may facilitate fluency by engaging the mirror neuronal system. In this experiment, three of the four experimental conditions demonstrated stuttering inhibition prior to speech production, yet the fluency enhancement derived from the passive observation of pseudostuttered speech is of highest importance, since it demonstrates both the possibility for passive stuttering inhibition prior to speech production and a compelling example of the link between speech perception and production's benefiting a pathological population. Emulators of choral speech that provide passive inhibition of stuttering (e.g., delayed auditory feedback or frequency-altered feedback) are immediate and effective stuttering inhibitors when provided simultaneously with speech production and may also engage mirror systems. However, these potent fluency enhancers could further benefit from supplementation by a preceding inhibitory agent when speech initiation is difficult, a characteristic common to many who stutter (Bloodstein, 1995). With the recognition of the role of gestural imitation, syllabic repetitions, and the mirror system in fluency enhancement, the link between speech perception and production may be further explored to provide functional clinical avenues for the treatment of this debilitating pathology.

\section{REFERENCES}

ADAMS, M. R., \& Moore, W. H. (1972). The effects of auditory masking on the anxiety level, frequency of dysfluency, and selected vocal characteristics of stutterers. Journal of Speech \& Hearing Research, 15, 572-578.

Ambrose, N. G., \& YAIRI, E. (1999). Normative disfluency data for early childhood stuttering. Journal of Speech, Language \& Hearing Research, 42, 895-909.

ARMSON, J., \& KALINOWSKI, J. (1994). Interpreting results of the fluent speech paradigm in stuttering research: Difficulties in separating cause from effect. Journal of Speech \& Hearing Research, 37, 69-82.

Blood, G. W., Blood, I. M., Bennett, S., Simpson, K. C., \& Susman, E. J. (1994). Subjective anxiety measurements and cortisol responses in adults who stutter. Journal of Speech \& Hearing Research, 37, 760768.

Bloodstein, O. (1995). A handbookon stuttering (5th ed.). San Diego: Singular.

CHERRY, E., \& SAYERS, B. (1956). Experiments upon total inhibition of stammering by external control and some clinical results. Journal of Psychosomatic Research, 1, 233-246.

CoHen, J. (1960). A coefficient of agreement for nominal scales. Educational \& Psychological Measurement, 20, 37-46.

Curio, G., Neuloh, G., Numminen, J., Jousmaki, V., \& Hari, R. (2000). Speaking modifies voice-evoked activity in the human auditory cortex. Human Brain Mapping, 9, 183-191.

Dayalu, V. N., Saltuklaroglu, T., Kalinowski, J., Stuart, A., \& RAstATter, M. P. (2001). Producing the vowel /a/ prior to speaking inhibits stuttering in English speaking adults. Neuroscience Letters, 306, $111-115$.

Fishman, H. C. (1937). A study of the efficacy of negative practice as a corrective for stammering. Journal of Speech Disorders, 2, 67-72.

FLEISS, J. L. (1981). Statistical methods for rates and proportions (2nd ed.). New York: Wiley.

Gray, B. B., \& BRutTEn, E. J. (1965). The relationship between anxiety, fatigue, and spontaneous recovery in stuttering. Behavior Research \& Therapy, 2, 251-259.

Healey, E. C., \& Howe, S. W. (1987). Speech shadowing characteristics of stutterers' diotic and dichotic conditions. Journal of Communication Disorders, 20, 493-506.

JansSEn, P., \& KraAimaAt, F. (1980). Disfluency and anxiety in stuttering and non-stuttering adolescents. Behavior Analysis \& Modification, 4, 116-126.

JoHnson, W. (1959). The onset of stuttering: Research findings and implications. Minneapolis: University of Minnesota Press.

Kalinowski, J., Dayalu, V. N., Stuart, A., Rastatter, M. P., \& RAMI, M. (2000). Stutter-free and stutter-filled speech signals and their role in stuttering amelioration for English speaking adults. Neuroscience Letters, 293, 115-118.

Kalinowski, J., \& Saltuklaroglu, T. (2003). Choral speech: The 
amelioration of stuttering via imitation and the mirror neuronal system. Neuroscience \& Biobehavioral Reviews, 27, 339-347.

Ke PPE L, G. (1991). Design and analysis: A researcher's handbook(3rd ed.). Upper Saddle River, NJ: Prentice-Hall.

Kohler, E., Keysers, C., Umiltà, M. A., Fogassi, L., Gallese, V., \& Rizzolatti, G. (2002). Hearing sounds, understanding actions: Action representation in mirror neurons. Science, 297, 846-848.

Kondas, O. (1967). The treatment of stammering in children by the shadow method. Behavior Research \& Therapy, 5, 325-329.

KraAimaAt, F. L., JansSen, P., \& VAN DAM-BAGgen, R. (1991). Social anxiety and stuttering. Perceptual \& Motor Skills, 72, 766.

Liberman, A. M., \& Mattingly, I. G. (1985). The motor theory of speech perception revised. Cognition, 21, 1-36.

MEISNER, J. H. (1946). Relation between voluntary non-fluency and the frequency of stuttering in oral reading. Journal of Speech Disorders, 11, 13-18.

Numminen, J., \& Curio, G. (1999). Differential effects of overt, covert and replayed speech on vowel-evoked responses of the human auditory cortex. Neuroscience Letters, 272, 29-32.

Numminen, J., SAlme Lin, R., \& HARI, R. (1999). Subject's own speech reduces reactivity of the human auditory cortex. Neuroscience Letters, 265, 119-122.

Peters, H. F. M., \& Hulstijn, W. (1984). Stuttering and anxiety: The difference between stutterers and nonstutterers in verbal apprehension and physiologic arousal during the anticipation of speech and nonspeech tasks. Journal of Fluency Disorders, 9, 67-84.
ReID, C. G., \& Lingwall, J. B. (1976). Some relationships between punishments, stuttering, and galvanic skin responses. Journal of Speech \& Hearing Research, 19, 197-205.

REID, C. G., \& LINGWALL, J. B. (1980). Conditioned stimulus effects on stuttering and GSRs. Journal of Speech \& Hearing Research, 23, 336343.

RITTERMAN, S. I., \& REIDENBACH, J. W. (1975). Inter-digital variability in the palmer sweat indices of adult stutterers. Journal of Fluency Disorders, 1, 33-46.

Rizzolatti, G., \& ARbiB, M. A. (1998). Language within our grasp. Trends in Neurosciences, 21, 188-194.

Saltuklaroglu, T., Dayalu, V. N., \& Kalinowski, J. (2002). Reduction of stuttering: The dual inhibition hypothesis. Medical Hypothesis, 58, 67-71.

SKoYles, J. R. (1998). Speech phones are a replication code. Medical Hypothesis, 50, 167-173.

VAN RIPER, C. (1973). The treatment of stuttering. Englewood Cliffs, NJ: Prentice-Hall.

WingATE, M. E. (1964). Recovery from stuttering. Journal of Speech \& Hearing Disorders, 29, 312-321.

(Manuscript received August 3, 2002;

revision accepted for publication July 10, 2003.) 